score using a KP Colorado (KPCO) cohort and the same patient characteristics. We calculated and plotted the observed one-year CDI risk for each decile of predicted risk for both cohorts. Results: The development cohort included 359,696 KPNW patients; 591 experienced CDI, a one-year incidence of 2.1 CDIs per 1000 patients. Twenty-two predictors of CDI were included in the risk score. Of these, age 60 years or greater, hospitalization of seven or more days within the previous 60 days, liver disease, and use of cephalosporins or clindamycin within the previous 60 days contributed to an approximate doubling of risk for CDI. The risk score separated high-risk patients from low-risk patients; the highest-risk patients were nearly 50 times more likely to have CDI (observed risk of 9.5 vs. $0.2 \mathrm{CDIs}$ per 1000 patients). For the validation cohort, we followed 296,550 KPCO patients; 620 experienced $\mathrm{CDI}$, a one-year incidence of $2.3 \mathrm{CDIs}$ per 1000 patients. The risk score validated successfully in Colorado, as it was able to discriminate the highest and lowest risk patients; predictions agreed closely with observed risk in each decile. Discussion: Our risk score successfully discriminated between patients at highest and lowest risk for CDI and provided predictions which agreed closely with observed CDI risk, making it a useful risk management tool for healthcare providers. Of importance, there was good calibration among the group of patients at the highest-risk for CDI, who are likely to benefit the most from clinician recognition of this risk.

Keywords: Clostridium difficile; Risk Score; Pharmacoepidemiology doi:10.3121/cmr.2012.1100.cc5-04

CC5-05:

Comparative Safety of Infliximab and Etanercept on the Risk of Serious Infections - Does the Association Vary By Patient Characteristics?

Darren $\mathrm{Toh}^{1}$; Lingling $\mathrm{Li}^{1}$; Leslie Harrold ${ }^{2}$; Elizabeth Bayliss ${ }^{3}$; Jeffrey Curtis $^{4}$; Liyan Liu ${ }^{5}$; Lang Chen ${ }^{4}$; Carlos Grijalva ${ }^{6}$; Lisa Herrinton ${ }^{5}$

${ }^{1}$ Harvard Pilgrim Health Care Institute, Harvard Medical School; ${ }^{2}$ Fallon Community Health Plan; ${ }^{3}$ Kaiser Permanente Colorado; ${ }^{4}$ University of Alabama at Birmingham; ${ }^{5}$ Kaiser Permanente Northern California; ${ }^{6}$ Vanderbilt University

Background/Aims: Infliximab, a chimeric monoclonal anti-TNF? antibody, has been found to increase the risk of serious infections compared with the TNF receptor fusion protein etanercept in some studies. It is unclear whether the risk varies by patient characteristics. We conducted a study to address this question. Methods: We identified members of Kaiser Permanente Northern California who initiated infliximab $(n=793)$ or etanercept $(n=2,692)$ in 19972007. Using a Cox model, we estimated the propensity score-adjusted hazard ratio (HR) and 95\% confidence interval (CI) of serious infections requiring hospitalization or opportunistic infections comparing infliximab with etanercept following treatment initiation. We estimated the stratum-specific HRs by age ( $<65$ vs. $>=65$ years), sex, race/ethnicity (Non- Hispanic White, African-American, Hispanic, Asian American, Native American, and other/ unknown), body mass index ( $<25 \mathrm{vs.}>=25 \mathrm{~kg} / \mathrm{m} 2$ ), and smoking status (non, past, and current smokers); and performed likelihood ratio tests to examine whether the HR differed by these patient characteristics. Results: The crude incidence rate of serious infections per 100 person-years was $5.4(95 \% \mathrm{CI}$ : $3.8,7.5)$ in patients $<65$ years and $16.0(10.4,23.4)$ in patients $>=65$ years during the first three months following treatment initiation. Compared with etanercept, the adjusted HR during this period was elevated for infliximab in patients $<65$ years (HR 3.01; 95\% CI: 1.49, 6.07), but not in those $>=65$ years (HR $0.94 ; 0.41,2.13)$. The test for homogeneity was marginally statistically significant ( $\mathrm{p}$-value $=0.06$ ). Findings did not suggest that the HR varied by other patient characteristics examined. Discussion: An increased risk of serious infections associated with infliximab relative to etanercept did not appear to be modified by patients' sex, race/ethnicity, body mass index, or smoking status. There was an indication that the increased risk might vary by age. Additional studies are warranted to verify or refute this finding.

Keywords: Pharmacoepidemiology; Autoimmune Disease doi:10.3121/cmr.2012.1100.cc5-05

\section{Prevention and Wellness}

PS2-33:

Non-physician Providers, Cancer Screening and Health Behavior Counseling

Deanna Kepka ${ }^{1}$; K. Robin Yabroff ${ }^{1}$

${ }^{1}$ National Cancer Institute

Background: Patients who receive a physician's recommendation for cancer screening and behavioral modification are most likely to comply with these recommendations. However, physicians face time constraints that make it nearly impossible to provide all recommended preventive services. Furthermore, the 2010 Affordable Care Act will expand health insurance coverage to 42 million Americans by 2014. This will increase demand for primary care. Non-physician providers may help meet this new demand for primary care and ensure compliance with preventive services recommendations. Methods: Data from the 2005 National Health Interview Survey were analyzed using multivariate logistic regression to assess the association between provider type seen in past 12 months and compliance with U.S. Preventive Services Task Force cancer screening recommendations and receipt of behavior counseling among age-eligible adults $(n=23,201)$. Models for each screening test were adjusted for age, level of education, and insurance status and stratified by gender. Results: About $15 \%$ of NHIS participants $(\mathrm{N}=4,652)$ saw a non-physician provider (nurse practitioner, certified nurse midwife or physician assistant) and a primary care physician. In adjusted analyses, age-eligible women were more likely to be compliant with Pap screening (OR: 5.0; 95\% CI: 4.2 - 5.9), mammography (OR: 6.6; $95 \%$ CI: $5.2-8.4$ ) and colorectal screening recommendations (OR: 7.8; $95 \%$ CI: $5.3-11.4$ ) if they saw a non-physician provider and primary care physician compared to not seeing any provider. Similarly, men were more likely to be compliant with colorectal screening recommendations (OR: 9.6; $95 \%$ CI: $6.9-13.5$ ) if they saw a non-physician provider and primary care physician. Women and men were more likely to report a provider asking about smoking status if they saw a non-physician provider and primary care physician than those who saw other types of healthcare providers ((OR: 2.2 ; 95\% CI: 2.0 - 2.4) and (OR: 3.0; 95\% CI: $2.4-3.7$ ), respectively). Conclusions: Seeing a non-physician provider and a primary care physician is related to an increased likelihood of compliance with cancer screening recommendations and receipt of health behavior counseling. Opportunities exist for non-physician providers to increase cancer screening and receipt of behavioral counseling during this era of healthcare reform.

Keywords: Non-Physician Providers; Cancer Screening; Prevention and Wellness

doi:10.3121/cmr.2012.1100.ps2-33

CB8-01:

Prevalence of Elevated Blood Pressure and BMI in U.S. Children and Adolescents

Patrick O'Connor'; Emily Parker'; Jay Desail'; David Magid ${ }^{2}$; Kenneth Adams $^{1}$; Karen Margolis ${ }^{1}$ Matthew Daley ${ }^{2}$; Alan Sinaiko ${ }^{3}$; Elyse Kharbanda ${ }^{1}$; Joan $\mathrm{Lo}^{4}$

${ }^{1}$ HealthPartners; ${ }^{2}$ Kaiser Permanente Colorado; ${ }^{3}$ Amplatz Children's Hospital; ${ }^{4}$ Kaiser Permanente Northern California

Background/Aims: To ascertain the prevalence and joint prevalence of elevated BMI and elevated BP in children and adolescents receiving clinical care, using electronic medical record (EMR) data. Methods: We studied BP and BMI percentiles (\%) of 79,838 U.S. subjects age 3 to 17 years with three or more eligible BP percentile measures from $1 / 1 / 2007$ to $12 / 31 / 2009$. We extracted data on age, diastolic and systolic BP, height, and weight from electronic medical records (EMR) and calculated BMI percentiles and BP percentiles using standard methods. Here we report by age group the proportions of subjects with (a) normal BMI and normal BP percentile, (b) elevated BMI $>=85$ th percentile or $>=95$ th percentile, or (c) hypertension (HT) defined as elevated BP $>=95$ th percentile on 3 or more occasions, or Pre-Hypertension (Pre-HT) defined as BP $>=90$ th percentile at least once but not meeting definition for HT. Results: Data Supplied as a Table, Not 